Laban, G. and Araujo, T. (2020) The Effect of Personalization Techniques in Users' Perceptions of Conversational Recommender Systems. In: 20th ACM International Conference on Intelligent Virtual Agents (IVA '20), Scotland, Virtual Event, UK, 20-22 Oct 2020, 34. ISBN 9781450375863.

There may be differences between this version and the published version. You are advised to consult the publisher's version if you wish to cite from it.

(C) The Authors 2020. This is the author's version of the work. It is posted here for your personal use. Not for redistribution. The definitive Version of Record was published in Proceedings of the 20th ACM International Conference on Intelligent Virtual Agents (IVA '20), Scotland, Virtual Event, UK, 20-22 Oct 2020, Article No.: 34. ISBN 9781450375863. http://dx.doi.org/10.1145/3383.52.3423890.

http://eprints.gla.ac.uk/225702/

Deposited on: 26 October 2020

Enlighten - Research publications by members of the University of Glasgow http://eprints.gla.ac.uk 


\section{The Effect of Personalization Techniques in Users' Perceptions of Conversational Recommender Systems}

\author{
Guy Laban* \\ Guy.Laban@glasgow.ac.uk \\ Institute of Neuroscience and Psychology, \\ University of Glasgow \\ Glasgow, United Kingdom
}

\author{
Theo Araujo \\ T.B.Araujo@uva.nl \\ Amsterdam School of Communication Research, \\ University of Amsterdam \\ Amsterdam, The Netherlands
}

\begin{abstract}
Conversational recommender systems provide users with individually tailored recommendations in a flowing dialogue. These require users to disclose information proactively or reactively for receiving personalized recommendations, which can trigger users' resistance to the platform and to the recommendations. Accordingly, this study examined the extent to which user-initiated and system-initiated recommendations provided by a conversational recommender system influenced users' perceptions of it. The results of an online experiment entail that when recommendations are system-initiated, as compared to user-initiated, users perceive to be in less control and perceive the system as riskier. Furthermore, the results stress that systems that provide user-initiated or systeminitiated recommendations do not differ in users' perceptions of anthropomorphism.
\end{abstract}

\section{CCS CONCEPTS}

- Human-centered computing $\rightarrow$ Empirical studies in $\mathrm{HCI}$ HCI theory, concepts and models; • Security and privacy $\rightarrow$ Social aspects of security and privacy; • Applied computing $\rightarrow$ Online shopping; Psychology.

\section{KEYWORDS}

Recommender Systems, Conversational Agents, Personalization, Chatbots, E-commerce, Anthropomorphism, Privacy

\section{INTRODUCTION}

Conversational recommender systems are artificially intelligent computer programs that provide users with personalized recommendations (i.e., individually tailored recommendations) by targeting individual needs and communicating in a flowing dialogue [5][17]. These agents are designed with cognitive architectures to communicate in a human-like way are often evaluated, perceived and described as such [1][14]. Accordingly, these are often reported as promoting users' engagement in online settings [5][17]. Conversational recommender systems provide recommendations that are initiated by either the user or the system. When recommendations are user-initiated, users consciously and explicitly disclose their preferences and reactively share parameters that are relevant for receiving a personal recommendation (e.g., answering questions or clicking checkboxes to filter recommendations according to specific parameters). Alternatively, when recommendations are systeminitiated, the system proactively personalizes a recommendation based on previously collected consumer data (e.g., recommendations that follow users' web-browsing behaviour) [3][20][22]. While personalization techniques can have positive persuasive implications, the necessity of disclosing information, reactively or proactively, can also trigger resistance among users, towards the recommendations provided and towards the system[3][13][19][21][22]. Hence, we are asking:

To what extent do users' perceptions of conversational recommender system differ when receiving user-initiated and system-initiated recommendations?

Users perceptions of agents are driven by their cognitive reconstruction, wherein their beliefs or expectations about an agent further shape perception and behaviour [7]. Moreover, previous studies demonstrate that people tend to disclose more to humans than to artificial agents while generally being aware of it [15][16]. Hence, the actions of conversational recommender systems that provide user-initiated recommendations correspond better to the actions of a human agent. Therefore, it is expected that (H1) conversational recommender systems that provide user-initiated recommendations, compared to system-initiated recommendations, will be perceived as more anthropomorphic.

Perceptions of risk and control are vital factors for organizations to adopt innovative online solutions and are fundamental in consumer evaluation [11][21]. Perceived risk is described as one's perceptions of concern, discomfort and/or anxiety from a specific situation or process [9], whereas perceived control refers to ones' internal attribution of control during a procedure (e.g., receiving a recommendation from a conversational recommender system) [18]. Both risk and control are considered as necessary channels for establishing a sense of certainty, confidence, and autonomy in information systems [6][12]. As users ascribe mental capacities to agents and evaluate these accordingly [10], it is expected that when receiving system-initiated recommendations, compared to user-initiated recommendations, from a conversational recommender system; one would perceive to have less control $(\mathrm{H} 2)$ and will perceive the system as riskier $(H 3)$.

\section{METHODS}

A two (user-initiated recommendations vs. system-initiated recommendations) between-subjects factors online experiment was conducted with 141 participants between the ages of 19 to 65 ( $M=38.11, S D=12.17,49 \%$ females $)$ that were recruited using Amazon Mechanical Turk (MTurk). The study received an ethics review board approval. The online experiment used an external Qualtrics page. The conversational recommender systems were embedded in an online chat format in the Qualtrics page. To control for the data quality collected on MTurk and narrow the sample frame, MTurk users were filtered based on their user score, native 

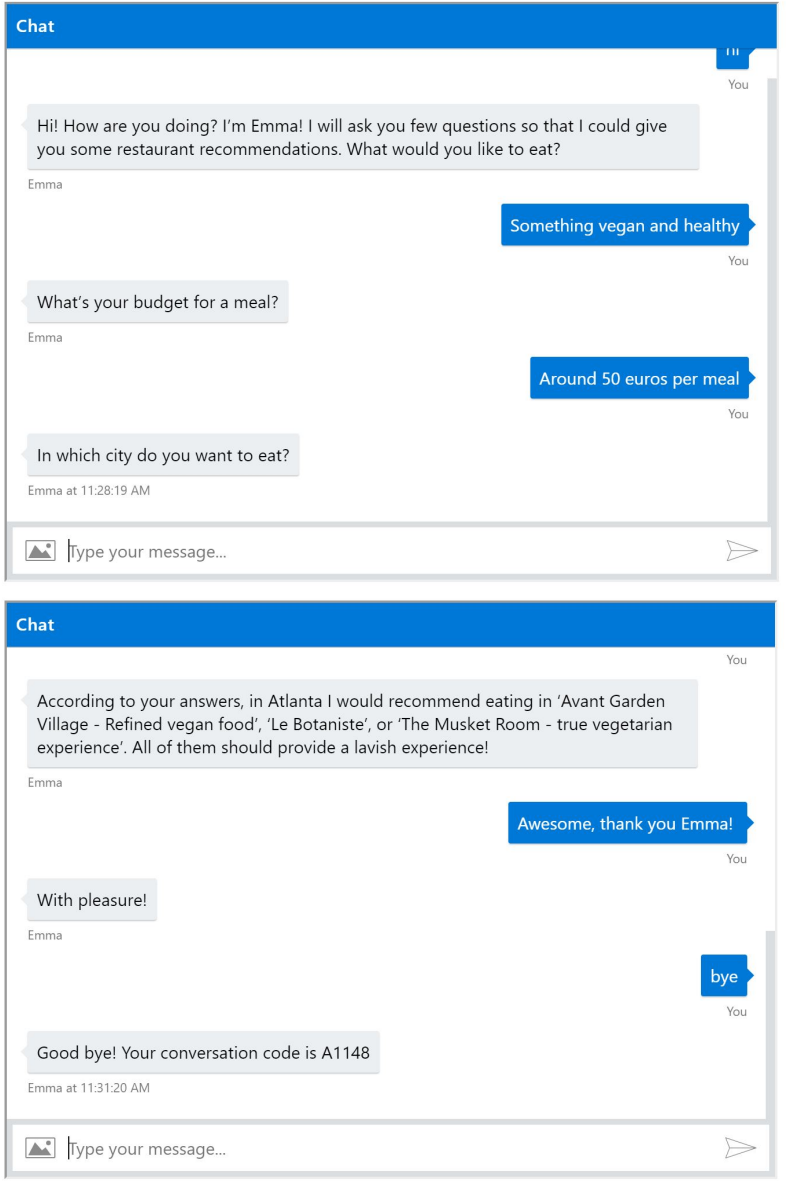

Figure 1: User-initiated recommendations condition.

language (being English) and geo-location (being within the United States). Moreover, throughout the online experiment, the participants had to answer two attention checks to validate that they are paying attention, and had to pass a "CAPTCHA" task to filter bots.

Participants were told that they are supposed to look for a restaurant using a conversational recommender system. The participants were randomly assigned to one of the two conversational recommender systems to receive a restaurant recommendation. In order to generate a personalized recommendation, the conversational recommender systems asked the participants three open-ended questions, asking them to disclose (1) their desired cuisine, (2) preferred budget for a meal, and (3) preferred location for a restaurant. The conversational recommender systems were created using the Conversational Agent Research Toolkit [2] especially for this study, and provide personalized recommendations based on the participants' answers. The systems contained pre-defined lists of values that corresponded to potential answers of the participants for providing recommendations. For example, pasta is an item in an Italian food list and "not much" is an item in a "small budget" list. Together the system can retrieve potential recommendations that answer to those values (i.e., an Italian restaurant for a small budget). The conversational recommender systems only differed in the source to

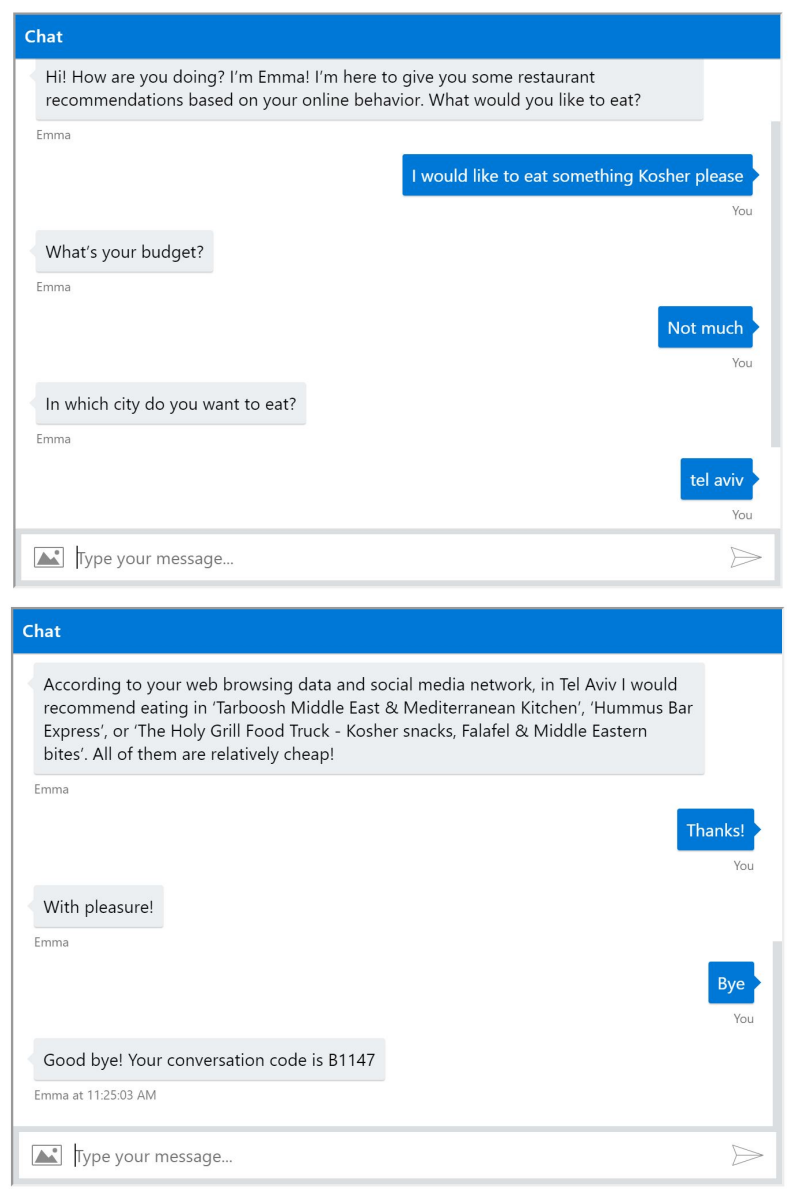

Figure 2: System-initiated recommendations condition.

which the recommendations were attributed to. In the user-initiated recommendations condition participants were explicitly informed by the conversational recommender system that they received recommendations based on their answers (i.e., "According to your answers..."; see Figure 1). In the system-initiated recommendations condition participants were explicitly informed by the conversational recommender system that they received recommendations based on their web browsing data and social media information (i.e., "According to your web browsing data and social media network..."; see Figure 2). Both systems were named Emma and communicated using first-person singular pronouns via online chat (see Figures 1 and 2).

After completing the task, participants evaluated the platform in terms of perceived anthropomorphism [4], control [18], and risk [8]. To account for the objectivity of the stimuli, participants were asked to evaluate how realistic they found the stimuli (i.e., the conversational recommender systems) to be on a seven-point Likertscale. An Independent sample T-Test demonstrates that there are no statistically significant differences in how realistic participants found the stimuli to be, between user-initiated $(M=5.55, S D=$ 1.36) and system-initiated $(M=5.06, S D=1.66)$ recommendations 
$($ Mdiff $=-.49, t(139)=-1.93, p=.056)$. After finishing their participation the participants were debriefed about the study.

\section{RESULTS}

Independent sample T-Tests were conducted to test the research's hypotheses. The results demonstrates that there are no statistically significant differences in perceived anthropomorphism between user-initiated $(M=4.89, S D=1.52)$ and system-initiated $(M=4.55, S D=1.58)$ recommendations $(M d i f f=-.34, t(139)=$ $-1.28, p=.201, d=.22)$. Hence, $H 1$ is rejected. Moreover, the results entail that users perceive to have more control $($ Mdiff $=.58$, $t(139)=-2.61, p=.010, d=.44)$ when receiving user-initiated $(M=4.21, S D=1.28)$ compared to system-initiated $(M=3.63$, $S D=1.37)$ recommendations. Therefore, $H 2$ is supported. Finally, users perceive conversational recommender systems that provide system-initiated $(M=4.32, S D=1.22)$ compared to user-initiated $(M=3.29, S D=1.40)$ recommendations, as riskier $(M$ diff $=1.03$, $t(139)=4.65, p<.001, d=.78)$. Hence, $H 3$ is supported.

\section{CONCLUSIONS}

While there are no differences in perceptions of anthropomorphism between the two systems, the two clearly differ in perceptions of risk and control. As users ascribed meaning to the conversation recommender system's actions, their perceptions followed the expected social norms of interpersonal relations. When disclosure was proactively initiated by the system, it was reflected in users' negative perceptions of the system. These results highlight the importance of conversational recommender systems sustaining positive moral mentality in their actions. This is especially important when considering the persuasive implications of personalized recommendations. The results of the study entail that when the system's actions and mentality conform to people's inherent expectations of it (i.e., inferring recommendations from information that was given willingly by the user), it has the potential to be perceived more positively. On the other hand, when the system's actions do not conform to these expectations (i.e., using users' personal information) it can result with the users demonstrating a sense of resistance to the system.

\section{REFERENCES}

[1] Theo Araujo. 2018. Living up to the chatbot hype: The influence of anthropomorphic design cues and communicative agency framing on conversational agent and company perceptions. Computers in Human Behavior 85 (2018), 183-189. https://doi.org/10.1016/j.chb.2018.03.051

[2] Theo Araujo. 2020. Conversational Agent Research Toolkit: An alternative for creating and managing chatbots for experimental research. Computational Communication Research 2, 1 (2020), 35-51. https://doi.org/10.5117/CCR2020.1. 002.ARAU

[3] Neeraj Arora, Xavier Dreze, Anindya Ghose, James D. Hess, Raghuram Iyengar, Bing Jing, Yogesh Joshi, V. Kumar, Nicholas Lurie, Scott Neslin, S. Sajeesh, Meng Su, Niladri Syam, Jacquelyn Thomas, and Z. J. Zhang. 2008. Putting one-to-one marketing to work: Personalization, customization, and choice. Marketing Letters 19, 3 (2008), 305. https://doi.org/10.1007/s11002-008-9056-z

[4] Christoph Bartneck, Dana Kulić, Elizabeth Croft, and Susana Zoghbi. 2009. Measurement Instruments for the Anthropomorphism, Animacy, Likeability, Perceived Intelligence, and Perceived Safety of Robots. International fournal of Social Robotics 1, 1 (2009), 71-81. https://doi.org/10.1007/s12369-008-0001-3

[5] Konstantina Christakopoulou, Filip Radlinski, and Katja Hofmann. 2016. Towards Conversational Recommender Systems. In Proceedings of the 22nd ACM SIGKDD International Conference on Knowledge Discovery and Data Mining (San Francisco, California, USA) (KDD '16). Association for Computing Machinery, New York, NY, USA, 815-824. https://doi.org/10.1145/2939672.2939746
[6] P. Cofta. 2007. Confidence, trust and identity. BT Technology fournal 25, 2 (2007), 173-178. https://doi.org/10.1007/s10550-007-0042-4

[7] Emily S Cross, Richard Ramsey, Roman Liepelt, Wolfgang Prinz, and Antonia de C Hamilton F. 2016. The shaping of social perception by stimulus and knowledge cues to human animacy. Philosophical transactions of the Royal Society of London.Series B, Biological sciences 371, 1686 (2016), 20150075. https://doi.org/10.1098/rstb.2015.0075

[8] Tamara Dinev, Heng Xu, Jeff H. Smith, and Paul Hart. 2013. Information privacy and correlates: an empirical attempt to bridge and distinguish privacy-related concepts. European fournal of Information Systems 22, 3 (05/01 2013), 295-316. https://doi.org/10.1057/ejis.2012.23 doi: 10.1057/ejis.2012.23.

[9] Grahame R. Dowling and Richard Staelin. 1994. A Model of Perceived Risk and Intended Risk-Handling Activity. Journal of Consumer Research 21, 1 (1994), 119-34. https://doi.org/10.1086/209386

[10] Nicholas Epley and Adam Waytz. 2010. Mind Perception. John Wiley and Sons Ltd. https://doi.org/10.1002/9780470561119.socpsy001014

[11] Mauricio S. Featherman and Paul A. Pavlou. 2003. Predicting e-services adoption: a perceived risk facets perspective. International fournal of Human-Computer Studies 59, 4 (2003), 451-474. https://doi.org/10.1016/S1071-5819(03)00111-3

[12] Ed Gerck. 2002. Trust as Qualified Reliance on Information, Part I. The COOK Report on Internet ISSN 1071 - 6327, Vol. X (01/10 2002), 19-24. https://doi.org/ 10.13140/RG.2.2.22646.04165

[13] Guy Laban and Theo Araujo. 2020. Don't Take it Personally: Resistance to Individually Targeted Recommendations with Anthropomorphic Recommender Systems. https://doi.org/10.31234/osf.io/w4mkv

[14] Guy Laban and Theo Araujo. 2020. Working Together with Conversational Agents: The Relationship of Perceived Cooperation with Service Performance Evaluations. In Chatbot Research and Design, Asbjørn Følstad, Theo Araujo, Symeon Papadopoulos, Effie Lai-Chong Law, Ole-Christoffer Granmo, Ewa Luger, and Petter Bae Brandtzaeg (Eds.). Springer International Publishing, Cham, 215228. https://doi.org/10.1007/978-3-030-39540-7_15

[15] Guy Laban, Jean-noël George, Val Morrison, and Emily S Cross. 2020. Tell Me More! Assessing Interactions with Social Robots From Speech. https: //doi.org/10.31234/osf.io/jkht2

[16] Guy Laban, Val Morrison, and Emily S Cross. 2020. Let's Talk About It! Subjective and Objective Disclosures to Social Robots. In Companion of the 2020 ACM/IEEE International Conference on Human-Robot Interaction. Association for Computing Machinery, Cambridge, UK, 328-330. https://doi.org/10.1145/3371382.3378252

[17] Lingyun Qiu and Izak Benbasat. 2009. Evaluating Anthropomorphic Product Recommendation Agents: A Social Relationship Perspective to Designing Information Systems. Fournal of Management Information Systems 25, 4 (2009), 145-182. https://doi.org/10.2753/MIS0742-1222250405

[18] Dan Russell. 1982. The Causal Dimension Scale: A measure of how individuals perceive causes. Journal of personality and social psychology 42, 6 (1982), 11371145. https://doi.org/10.1037/0022-3514.42.6.1137

[19] S Shyam Sundar. 2020. Rise of Machine Agency: A Framework for Studying the Psychology of Human-AI Interaction (HAII). Fournal of Computer-Mediated Communication 25, 1 (01 2020), 74-88. https://doi.org/10.1093/jcmc/zmz026

[20] S. S. Sundar and Sampada S. Marathe. 2010. Personalization versus Customization: The Importance of Agency, Privacy, and Power Usage. Human Communication Research 36, 3 (2010), 298-322. https://doi.org/10.1111/j.1468-2958.2010.01377.x

[21] Cong Wang, Yifeng Zheng, Jinghua Jiang, and Kui Ren. 2018. Toward PrivacyPreserving Personalized Recommendation Services. Engineering 4, 1 (February 2018 2018), 21-28. https://doi.org/10.1016/j.eng.2018.02.005

[22] Bo Zhang and S. Shyam Sundar. 2019. Proactive vs. reactive personalization: Can customization of privacy enhance user experience? International fournal of Human-Computer Studies 128 (2019), 86 - 99. https://doi.org/10.1016/j.ijhcs.2019. 03.002 